\title{
Assessment of awareness levels toward colorectal cancer early detection and prevention in Saudi Arabia
}

\begin{abstract}
Background: Awareness of healthy individuals with colorectal cancer (CRC) can identify segments of the population that would most benefit from targeted education programs. Therefore, the aim of the present study was to assess the levels of awareness towards early colonoscopy screening and surgical management of colorectal cancer in the Kingdom of Saudi Arabia (KSA)
\end{abstract}

Methodology: This study randomly surveyed 1217 individuals, their age ranging from 20 to 70 years with a mean age of 36 years. An anonymous online questionnaire was used for data collection.

Results: The sample was consisted of 630/1217(51.5\%) women and 587/1217(48.5\%) men. Among the study population, 591/1217 (48.6\%) think that people over the age of 50 years are more likely to develop colorectal cancer, $751 / 1217(61.9 \%)$ think that the lack of exercise is a contributing factor for colorectal cancer, similarly $823 / 1217(67.9 \%)$ think that the style of food and eating red meat can contribute to colorectal cancer. The majority of respondents affirm the benefits of CRC screening: 1067/1217 (88\%) think that screening using endoscopy contributes to the early detection of colorectal cancer and 1075/1217 (88.8\%) think that early endoscopy contributes to reduce the complications of colorectal cancer.

Conclusion: The awareness level of CRC among Western Province population of Saudi Arabia is relatively lower in some parts, and therefore, the implementation of awareness programs may improve the awareness of CRC.

Keywords: colorectal cancer, colonoscopy, Saudi Arabia, early detection
Volume 6 Issue 3 - 2017

\author{
Bassam Ahmed Almutlaq,' Bayan Esmaeel \\ Bokhari, ${ }^{2}$ Hesham Hussain Alharbi,' Ahmad \\ soud alzayed,' Ayman sayed abouhamda, ${ }^{3}$ \\ Majid Zannon Alturkstani, ${ }^{4}$ Abdulghani \\ Abdulaziz Alzamzami, ${ }^{3}$ Yousef Ehab Jan, ${ }^{3}$ \\ Saeed Ahmed Alzahrani,3 Abdulrahman \\ Abdullah Takrooni, ${ }^{3}$ Hussain Gadelkarim \\ Ahmed' \\ 'University of Hail, Saudi Arabia \\ ${ }^{2}$ National Guard Hospital, Saudi Arabia \\ ${ }^{3}$ Ibn sina National College for Medical Studies, Saudi Arabia \\ ${ }^{4}$ Batterjee Medical College, Saudi Arabia
}

Correspondence: Prof. Hussain Gadelkarim Ahmed, University of Hail-College of medicine 2440, Saudi Arabia, Email hussaingad1972@yahoo.com

Received: December 28, 2016 | Published: March 03, 2017

\section{Introduction}

Colorectal cancer $(\mathrm{CRC})$ is one of the commonest cancers with an estimated incidence of 72,090 males and 70,480 females and a cause of specific death of 51,370 in $2010 .{ }^{1}$ CRC is a multifactorial illness resulting from lifestyle, genetic, and environmental factors. There are hereditary and non-hereditary CRC types; though, the bulk is nonhereditary and mostly caused by somatic mutations in response to environmental factors. In previous years, scientists have concentrated their attention on the mechanisms behind these factors and the approaches of enhancing disease prevention and treatment. Enhancing the awareness of the population with respect to the advantages of a healthy lifestyle, comprising a balanced diet accompanying with exercise, could totally diminish CRC risk. ${ }^{2}$

The choice to participate in CRC screening relies on a person's awareness CRC screening. Awareness influenced assessments of cancer, attitudes towards CRC screening approaches, and motivation for screening. Factors mediating awareness counted in public education to target misconceptions, primary care physician efforts to endorse screening, and the inspiration of friends and family. Particular barriers to participation in populations with poorer participation rates included language barriers, logistical challenges to joining screening tests, and cultural beliefs. ${ }^{3}$

The fecal occult blood test is widely used for CRC screening in several countries and its effectiveness in reducing CRC incidence and mortality has been demonstrated; however, the low participation rate in CRC screening programs is a problem to be solved in every country. ${ }^{4}$ Improvement in the public awareness of CRC and promotion of CRC screening by physicians will help to raise the participation rate and reduce the number of deaths caused by CRC. Regarding screening colonoscopy, several studies have recently demonstrated its effectiveness in reducing CRC incidence and mortality. However, at present, CRC screening colonoscopy is not adopted as a primary population-based screening tool because of staffing constraints in relation to large population sizes, increased medical costs. ${ }^{5}$

$\mathrm{CRC}$ is one of the commonest cancers and a prime cause of cancer mortality in the Kingdom of Saudi Arabia (KSA). CRC in KSA is often diagnosed at progressive stages with metastases and is linked to poor prognosis and short survival. Countrywide awareness campaigns and screening programs for CRC are essential for prevention, early detection and sufficient management of CRC. ${ }^{6}$ Therefore, the aim of the present study was to assess the levels of awareness towards toward early colonoscopy screening and surgical management of colorectal cancer in Saudi Arabia.

\section{Materials and methods}

This is a community survey involved 1217 volunteers living in the city of Jeddah, Kingdom of Saudi Arabia (KSA). All study subjects were randomly selected regardless to gender or sex. A self-administered online questionnaire was used for data collection including: Demographic characteristics (age, gender, education level, and income). Opinions about Colorectal cancer (CRC) assessment 
including 7 questions. A score of 1 was given to yes and 0 otherwise. For each subject, a maximum score of 7 was calculated. A scoring system was applied to measure the respondents' beliefs towards CRC. The knowledge score was categorized into two levels indicated by poor knowledge (0-2), average knowledge (3-4) and good knowledge (5-7). Knowledge about early endoscopy assessment including 3 questions. A score of 1 was given to yes and 0 otherwise. For each subject, a maximum score of 3 was calculated. A scoring system was applied to measure the respondents' knowledge about early endoscopy. The knowledge score was categorized into 2 levels indicated by weak knowledge (0-1) and good knowledge (2-3).

a. Ethical consent: An informed consent was obtained from the participants included in this research before filling the questionnaire.

b. Statistical analysis: Data were entered into the Statistical Package for Social Sciences (SPSS, version 20) and descriptive analysis conducted. Association of respondents' characteristics with beliefs about CRC and knowledge about early endoscopy as a prevention tool was evaluated using: Frequencies and percentages, Chi-squared test. Statistical significance was accepted at $\mathrm{p}<0.05$.

\section{Results}

In this study awareness toward CRC was assessed among 1217 Saudi, their ages ranging from 18 to 70 with mean age of 36 years. The response rate was $96.3 \%$. Out of 1217 participants, 630/1217 $(51.8 \%)$ were males and 587/1217 (48.2\%) were females (Figure 1).

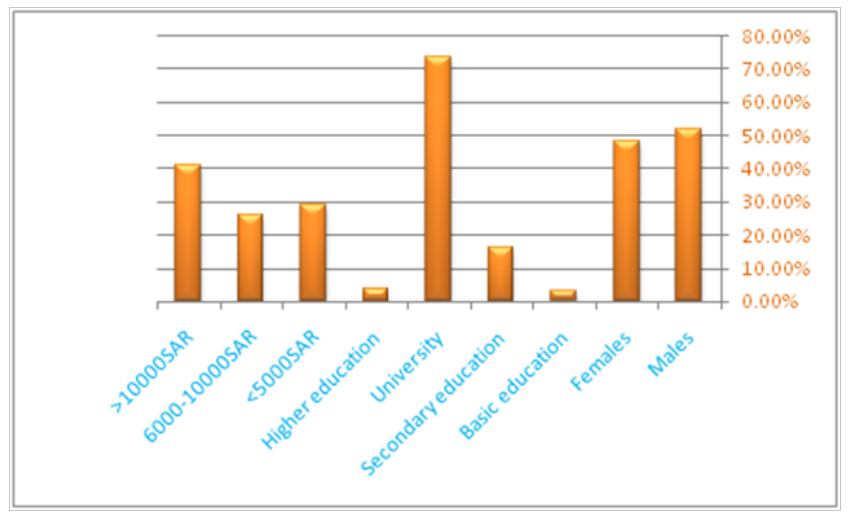

Figure I Description of the study population by demographical characteristics.

In regard to the education, $898 / 1217(73.8 \%$ ) of the respondents have a university's degree, followed by diploma, high school level, $\mathrm{Ph} . \mathrm{D}$, secondary school level and Primary school level representing $197 / 1217$ (16.2\%), 82/1217 (6.7\%), 22/1217 (1.8\%), 11/1217(0.9\%) and $5 / 1217(0.41 \%)$, respectively as shown in Figure 1. In regard to income, $501(42.9 \%)$ of the respondents have an income of $(>2.666$ US \$), $252(21.6 \%)$ have an income of (<800 US \$), $213(18.2 \%)$ with an income between (1.866-2.666 US \$), 103(8.8\%) have an income between (1.333-1.866 US \$), and 99(8.5\%) have an income between (1.333-1.866 US \$) as shown in Figure 1.

Table 1, Figure 2 Summarize the responses to questions (Q1-Q10) on knowledge regarding colorectal cancer. The results show that the majority of respondents $(38.5 \%)$ think that age is one of the factors of colorectal cancer, followed by $32.8 \%$ of the respondents who think not that age is one of the factors of colorectal cancer. Then $28.7 \%$ of the respondents seemed to be not sure if age is one of the factors of colorectal cancer, as indicated in Table $1 \&$ Figure 2. It shows that there are statistically significant differences in the responses of respondents to the section on "Do you think that age is one of the factors of colorectal cancer" attributed to the age $(\mathrm{F}=6.671, \mathrm{p}$ value $=0.001<0.05$ ). The null hypothesis (equality of the mean of age within each of the 3 groups) is clearly rejected ( $\mathrm{p}=0.001)$.

The results show that the majority of respondents (48.6\%) think that people over the age of 50 years are more likely to develop colorectal cancer, followed by $36.2 \%$ of the respondents who seemed to be not sure if people over the age of 50 years are more likely to develop colorectal cancer or not. Then $15.2 \%$ of the respondents think not that people over the age of 50 years are more likely to develop colorectal cancer, as indicated in Table $1 \&$ Figure 2. It shows that there are no statistically significant differences in the responses of respondents to the section on "People over the age of 50 years are more likely to develop colorectal cancer" attributed to the age $(\mathrm{F}=$ 2,760 , $p$ value $=0.064>0.05$ ).

The results show that the majority of respondents $(61.9 \%)$ think that the lack of exercise of the contributing factors of colorectal cancer, followed by $22.9 \%$ of the respondents who seemed to be not sure if the lack of exercise of the contributing factors of colorectal cancer. Then $15.2 \%$ of the respondents think not that the lack of exercise of the contributing factors of colorectal cancer, as indicated in Table $1 \&$ Figure 2.

The results show that the majority of respondents $(67.9 \%)$ think that the style of food and eating red meat can contribute to colorectal cancer factors, followed by $21,0 \%$ of the respondents who seemed to be not sure if the style of food and eating red meat can contribute to colorectal cancer factors or not. Then $11.1 \%$ of the respondents think not that the style of food and eating red meat can contribute to colorectal cancer factors, as indicated in Table $1 \&$ Figure 2.

The results show that the majority of respondents $(52.3 \%)$ think that genetics play a role in colorectal cancer, followed by $24.3 \%$ of the respondents who think not that genetics play a role in colorectal cancer. Then $23.4 \%$ of the respondents seemed to be not sure if genetics play a role in colorectal cancer or not, as indicated in Table 1 $\&$ Figure 2. It shows that there are statistically significant differences in the responses of respondents to the section on "Do you think that genetics play a role in colorectal cancer" attributed to the age $(\mathrm{F}=6.507$, $\mathrm{p}$ value $=0,002<0.05)$.

The results show that the majority of respondents $(65.4 \%)$ think that smoking is one of colorectal cancer factors, followed by $20.9 \%$ of the respondents who seemed to be not sure if smoking is one of colorectal cancer factors. Then $13.7 \%$ of the respondents think not that smoking is one of colorectal cancer factors, as indicated in Table $1 \&$ Figure 2.

The results show that the majority of respondents $(60.7 \%)$ think that mental illnesses like thinking and anxiety lead to obesity, which may increase the risk of CRC, followed by $31.6 \%$ of the respondents who seemed to be not sure if mental illnesses like thinking and anxiety lead to obesity. Then $7.7 \%$ of the respondents think not that mental illnesses like thinking and anxiety lead to obesity, as indicated in Table $1 \&$ Figure 2 . It shows that there are statistically significant differences in the responses of respondents to the section on "Do you think that there are diseases may cause or lead to colorectal cancer" attributed to the age $(\mathrm{F}=8.129$, $\mathrm{p}$ value $=0,000<0.05)$. The null hypothesis (equality of the mean of age within each of the 3 groups) is clearly rejected $(\mathrm{p}=0.000)$. 
Table I Responses to questions on knowledge regarding colorectal cancer

\begin{tabular}{|c|c|c|c|}
\hline Questions & No & Yes & Don't Know \\
\hline QI:Do you think that age is one of the factors of colorectal cancer & 398 & 467 & 348 \\
\hline Q2: People over the age of 50 years are more likely to develop colorectal cancer & 185 & 591 & 440 \\
\hline Q3: Do you think the lack of exercise of the contributing factors of colorectal cancer & 185 & 751 & 278 \\
\hline Q4: Do you think the style of food and eating red meat can contribute to colorectal cancer factors & 134 & 823 & 255 \\
\hline Q5: Do you think that genetics play a role in colorectal cancer & 295 & 634 & 284 \\
\hline Q6: Do you think that smoking is one of colorectal cancer factors & 166 & 793 & 253 \\
\hline Q7: Do you think that there are diseases may cause or lead to colorectal cancer & 93 & 735 & 382 \\
\hline $\begin{array}{l}\text { Q8: Do you think that screening using endoscopy contribute to the early detection of colorectal } \\
\text { cancer }\end{array}$ & 13 & 1067 & I33 (I I\%) \\
\hline Q9: Do you think it must be an annual survey checks for people above 50 years & 93 & 948 & 175 \\
\hline Q10: Do you think that early endoscopy contributes to reduce the complications of colorectal cancer & 24 & 1075 & 111 \\
\hline
\end{tabular}

The results show that the majority of respondents $(88.0 \%)$ think that screening using endoscopy contribute to the early detection of colorectal cancer, followed by $11,0 \%$ of the respondents who seemed to be not sure if screening using endoscopy contribute to the early detection of colorectal cancer or not. Then $1,1 \%$ of the respondents think not that screening using endoscopy contribute to the early detection of colorectal cancer, as indicated in Table $1 \&$ Figure 2.

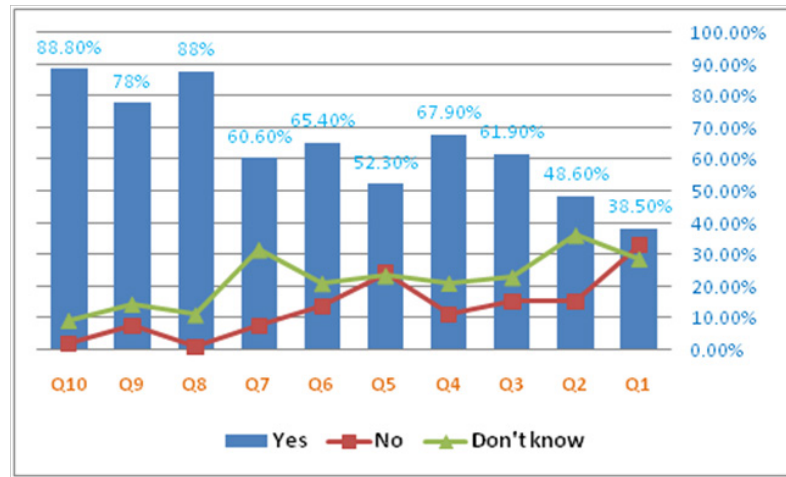

Figure 2 Responses to questions on knowledge regarding colorectal cancer.

The results show that the majority of respondents $(78,0 \%)$ think that it must be an annual survey checks for people above 50 years, followed by $14,4 \%$ of the respondents who seemed to be not sure if it must be an annual survey checks for people above 50 years or not. Then $7.6 \%$ of the respondents think not that it must be an annual survey checks for people above 50 years, as indicated in Table $1 \&$ Figure 2. The results show that the majority of respondents $(88.8 \%)$ think that early endoscopy contributes to reduce the complications of colorectal cancer, followed by $9.2 \%$ of the respondents who seemed to be not sure if early endoscopy contributes to reduce the complications of colorectal cancer or not. Then $2.0 \%$ of the respondents think not that early endoscopy contributes to reduce the complications of colorectal cancer, as indicated in Table $1 \&$ Figure 2.

On asking them about the treatment of colorectal cancer the majority of respondents $(72.5 \%)$ think that the treatment for colorectal cancer is surgical, followed by $16.2 \%$ of the respondents who think that the treatment for colorectal cancer is chemotherapy. Then $11.3 \%$ of the respondents think that the treatment for colorectal cancer is radiotherapy, as indicated in Figure 3. It shows that there are no statistically significant differences in the responses of respondents to the section on "In your opinion what is the treatment for colorectal cancer" attributed to the age $(\mathrm{F}=2.444$, $\mathrm{p}$ value $=0.087>0.05)$. On asking them, what are the survival ratio for after 5 years of the early diagnosis of colorectal cancer?, the majority of respondents $(49.5 \%)$ do not know what are the survival ratio for after 5 years of the early diagnosis of colorectal cancer, followed by $21.7 \%$ of the respondents who think that the survival ratio for after 5 years of the early diagnosis of colorectal cancer is $90 \%$. Then $17.4 \%$ of the respondents think that the survival ratio for after 5years of the early diagnosis of colorectal cancer is $70 \%$, and $8.6 \%$ of the respondents who think that the survival ratio for after 5 years of the early diagnosis of colorectal cancer is $40 \%$. Finally $2.8 \%$ of the respondents think that the survival ratio for after 5 years of the early diagnosis of colorectal cancer is $20 \%$, as shown in Figure 4.

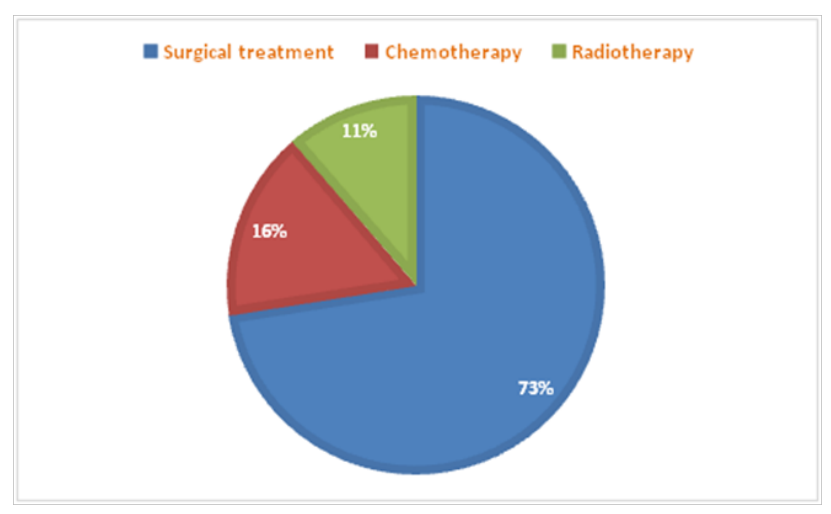

Figure 3 Describe the opinion on what is the treatment for colorectal cancer.

\section{Discussion}

Early detection of cancer significantly raises the likelihoods for successful overall management of patients. The most important components of early detection of CRC are education to encourage early diagnosis and screening. Improved awareness of potential threatening signs of CRC, amongst physicians, nurses and other health care providers as well as among the general public, can have a great influence on the CRC control. 


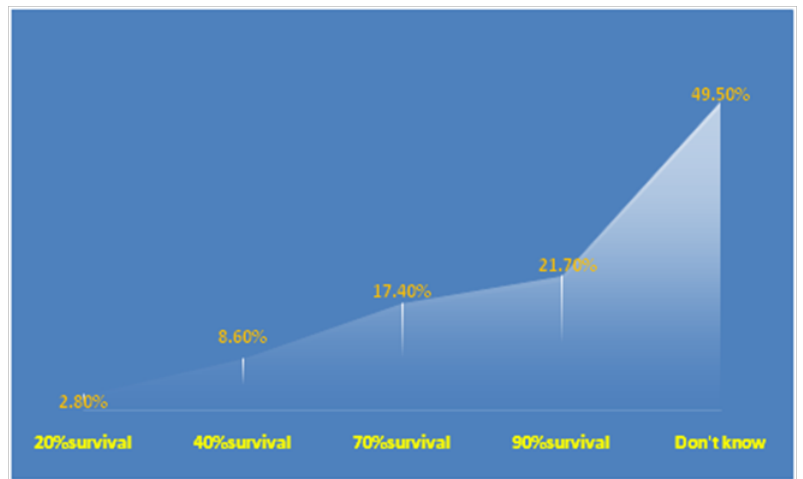

Figure 4 Describe the opinion on what are the survival ratio for after 5 years of the early diagnosis of colorectal cancer?

In the present study we tried to assess the general Saudi population awareness toward CRC. With regard to the question "Do you think that age is one of the factors of colorectal cancer", only $38.5 \%$ think that age is risk factor for CRC. These findings indicate a poor awareness level, since the relationship between age and CRC was well established in several studies. ${ }^{7-9}$ Increasing the cutoff screening age reduces the numbers of patients who undergo colonoscopy evaluation in CRC screening programs. ${ }^{10}$ When specified the age to over 50years in the present about $48.6 \%$ thought that people over the age of 50 years are more likely to develop colorectal cancer. However, around $78 \%$ ) thought that it must be an annual survey checks for people above 50 years.

In the present study about $61.9 \%$ of the participants thought that the lack of physical activities is a risk factor for colorectal cancer This percentage is relatively not so high. Physical activities have been found to reduce the risk of CRC. The reverse relationship between physical activities and CRC is constant. ${ }^{11}$ The rate, duration and intensity of physical activities are essential components of a public health message to reduce risk of CRC via practicing of physical activity. On the other hand, difficulties in assessing the precise amount of physical activity required and frequency and intensity of activity result in only rough approximations of dose wanted for a protective influence. Considerable amount of the literature propose that more intense activity is required to reduce CRC risk and that wherever between 3.5 and 4 hours of vigorous physical activities per week may be necessary to improve protection. Numerous biological processes have been suggested to clarify the relationship between physical activities and CRC; several of these processes also support the opinion that intense activities are most protective. Biological mechanisms such as: physical activities increasing gut motility; improving the immune system; reducing insulin and insulin-like growth factor levels; reducing obesity; increasing free radical scavenger systems; and influencing prostaglandin levels. Approximately $12-14 \%$ of CRC could be attributed to lack of frequent contribution in vigorous physical activity. ${ }^{12}$

About $67.9 \%$ of the participants thought that the style of food and eating red meat can contribute to $\mathrm{CRC}$ risk. However, this is relatively higher percentage indicating some sorts of awareness among the study population. Meat has been categorized by International Agency for Research on Cancer (IARC) as carcinogenic to humans. Numerous mechanisms for mutagenic properties of meat eating have been recognized but it is not clear, which cause cancer in humans. The scope to which whole abstention from meat protects against cancer is also unclear. ${ }^{13}$ A recent investigation by the World Health Organisation (WHO) has found that the consumption of processed meat and potentially red meat promotes carcinogenesis and can increase the risk of colorectal cancer. Processed meat is more carcinogenic compared to red meat because of the abundance of potent nitrosyl-heme molecules that form N-nitroso compound..$^{14}$

Approximately $52.3 \%$, thought that genetic risk factors play a role in CRC. It was found that about 3-5\% of CRCs are due to inherited genetic mutations. ${ }^{15}$ Genome-wide association studies have identified 17 germline single nucleotide polymorphisms (SNPs) significantly associated with incident CRC. ${ }^{16}$

A round $65.4 \%$ thought that smoking is one of colorectal cancer factors. Smoking has been implicated in many malignant diseases, but its association with colorectal cancer (CRC) is controversial. Some studies have shown that smoking was associated with a significantly increased risk of CRC. The associated risk was higher for men and for rectal cancers. The association of tobacco consumption and CRC risk appeared to be dose-related. ${ }^{17}$

About $60.7 \%$ of the participants thought that mental illnesses like thinking and anxiety lead to obesity, which may increase the risk of CRC. The causal relationship from obesity to anxiety disorders and vice versa is still under investigation. Pharmacological factors used for obesity treatment, such as rimonabant,were associated with depression and anxiety. Questions still remain regarding the role of obesity severity and subtypes of anxiety disorders. Besides, it is well known that in the morbidly obese patients before undergoing surgical treatment, unusual prevalence of psychopathology, namely depression and anxiety disorders, is observed. ${ }^{18}$

There is strong evidence that modifiable lifestyle factors such as obesity play a key role in colorectal carcinogenesis. Epidemiologic data have consistently reported a positive association between obesity and colorectal cancer. The relative risk associated with general obesity (as assessed by BMI) is higher in men than in women and for cancer of the colon than for cancer of the rectum. Abdominal obesity (as assessed by waist circumference or waist-to-hip ratio) is associated with an increased risk of colorectal cancer in both sexes, with stronger associations for cancer of the colon than for cancer of the rectum. ${ }^{19}$ Plausible biological mechanisms include insulin resistance, hyperinsulinemia, chronic inflammation, altered levels of growth factors, adipocytokines and steroid hormones. In addition to its effect on colorectal cancer incidence, obesity may play a role in colorectal cancer recurrence, treatment outcomes and survival. ${ }^{20,21}$ The adipokines adiponectin and leptin and adipocyte-mediated chronic low-grade inflammation represented by the acute-phase C-reactive protein may explain a substantial part of the association between obesity and risk of colorectal cancer. ${ }^{22}$

A bout $88.0 \%$ of the study subjects believe that screening using endoscopy contribute to the early detection of colorectal cancer. Highquality colonoscopy is needed to reduce the morbidity and mortality of colorectal cancer. Full-spectrum endoscopy has recently shown potential in improving adenoma detection during colonoscopy. ${ }^{23}$ Miss rate of polyps has been shown to be substantially lower with full-spectrum endoscopy (FUSE) compared with standard forwardviewing (SFV) colonoscopy in a tandem study at per polyp analysis. However, there is uncertainty on whether FUSE is also associated with a higher detection rate of colorectal neoplasia, especially advanced lesions, in per patient analysis. ${ }^{24}$ However, about $88.8 \%$ thought that early endoscopy contributes to the reduction of the complications of colorectal cancer. 
Several studies have conducted in this context from Saudi Arabia. A recent study to explore the knowledge and awareness about colorectal cancer (CRC) among undergraduate students of one of the leading universities in Saudi Arabia, along with the mode of information access. The study concluded that knowledge and awareness of students about CRC were not up to the mark. Medical students and female students had better knowledge in a few areas, but the overall situation is dismal. ${ }^{25}$ Another study investigated colorectal cancer (CRC) awareness in healthy individuals in Saudi Arabia in order to identify segments of the population that would most benefit from targeted education programs. The study concluded that although older individuals and those with higher education tended to answer questions correctly more often, there were some misconceptions regarding universally accepted screening protocols, symptoms, and general understanding of CRC in Saudi Arabia. A national education/ screening program in Saudi Arabia is recommended to improve CRC knowledge. ${ }^{26}$

Furthermore, several similar surveys have been conducted in Saudi Arabia. In a survey included 1070 participants most respondents believe that screening for colon cancer should begin at symptom onset $(42.9 \%)$. Less than $20 \%$ of all respondents believe that polyps are a risk factor for $\mathrm{CRC}$, which varied significantly according to level of education; however, even the most educated answered correctly less than $50 \%$ of the time. Similarly, only $34.8 \%$ of all respondents knew that a family history of CRC imparted a personal risk for CRC. ${ }^{27}$ In another study included 371 participants, of whom 52\%, 86\% and $60 \%$, didn't hear about early screening of CRC didn't perform screened of CRC and didn't know where to screen for colorectal cancer, respectively. The subjective assessment of the participants' Level of knowledge about colorectal cancer revealed poor, good and excellent in $53 \%, 37 \%$ and $10 \%$ of the respondents, respectively. Highest percentage for education level about Public awareness for CRC universal by $45 \%$ and lowest unlettered by $4 \%$ which varied significantly according to level of education. About 6 from 11 diagnosed with CRC and have a family history of CRC. ${ }^{28}$

Although, the questionnaire used in the present study was comprehensive regarding with regard to awareness associated factors, but many variables might be answered subjectively, due some community barriers.

\section{Conclusion}

The awareness level of CRC among Western Province is relatively lower in some parts, and therefore, the implementation of awareness programs may improve the awareness of CRC through continuous education programs, local media, or campaigns to encourage the early detection CRC.

\section{Acknowledgments}

None.

\section{Conflicts of interest}

The authors declare there is no conflict of interests.

\section{Funding}

None.

\section{References}

1. Kanna S, Ali A, Anna K, et al. Physician's awareness and practices toward colorectal cancer screening guidelines. J Clin Oncol. 2012;30(4_ suppl):431.
2. Aran V, Victorino AP, Thuler LC, et al. Colorectal Cancer: Epidemiology, Disease Mechanisms and Interventions to Reduce Onset and Mortality. Clin Colorectal Cancer. 2016;15(3):195-203.

3. Honein-AbouHaidar GN, Kastner M, Vuong V, et al. Systematic Review and Meta-study Synthesis of Qualitative Studies Evaluating Facilitators and Barriers to Participation in Colorectal Cancer Screening. Cancer Epidemiol Biomarkers Prev. 2016;25(6):907-917.

4. Schreuders EH, Grobbee EJ, Spaander MCW, et al. Advances in Fecal Tests for Colorectal Cancer Screening. Curr Treat Options Gastroenterol. 2016;14(1):152-162.

5. Sano Y, Byeon JS, Li XB, et al. Colorectal cancer screening of the general population in East Asia. Dig Endosc. 2016;28(3):243-249.

6. Aldiab A, Al Khayal KA, Al Obaid OA, et al. Clinicopathological features and predictive factors for colorectal cancer outcome in the Kingdom of Saudi Arabia. Oncology. 2016;92(2):75-86.

7. Lonardi S, Stefani M, Jirillo A, et al. Benefit of fluorouracil and folinic acid adjuvant in colon cancer elderly patients. J Clin Oncol. 2006;24(18_ suppl):13564.

8. Resch G, Poetscher M, Schauer W, et al. Colorectal cancer in elderly patients: A single-center experience. J Clin Oncol. 2011;29(4 suppl):576.

9. Koretz RL. Evidence-Based Guideline: The USPSTF recommends screening for colorectal cancer in adults 50 to 75 years of age. Ann Intern Med. 2016;165(6):JC26.

10. Wieten E, Schreuders EH, Nieuwenburg SA, et al. Effects of Increasing Screening Age and Fecal Hemoglobin Cutoff Concentrations in a Colorectal Cancer Screening Program. Clin Gastroenterol Hepatol. 2016;14(12):1771-1777

11. Brown JC, Winters-Stone K, Lee A, et al. Cancer, Physical Activity, and Exercise. Comprehensive Physiology. 2012;2(4):2775-2809.

12. Slattery ML. Physical activity and colorectal cancer. Sports Med. 2004;34(4):239-252.

13. Johnson IT. The cancer risk related to meat and meat products. $\mathrm{Br}$ Med Bull. 2016. 18 p.

14. Jeyakumar A, Dissabandara L, Gopalan V. A critical overview on the biological and molecular features of red and processed meat in colorectal carcinogenesis. J Gastroenterol. 2016.

15. Jenab-Wolcott J, Tan K, Heitjan DF, et al. Evaluation of physician knowledge and referral practices for colorectal cancer (CRC) genetic risk assessment: The experience at the Hospital of University of Pennsylvania (HUP). J Clin Oncol. 2011;29(4_suppl):379.

16. Phipps A, Garcia-Albeniz X, Hutter C, et al. Known colorectal cancer susceptibility loci and survival after colorectal cancer diagnosis. J Clin Oncol. 2012;30(4_suppl):394.

17. Tsoi KK, Pau CY, Wu WK, et al. Cigarette smoking and the risk of colorectal cancer: a meta-analysis of prospective cohort studies. Clin Gastroenterol Hepatol. 2009;7(6):682-688.

18. Lykouras L, Michopoulos J. Anxiety disorders and obesity. Psychiatriki. 2011;22(4):307-313.

19. Taylor RW, Jones IE, Williams SM, et al. Evaluation of waist circumference, waist-to-hip ratio, and the conicity index as screening tools for high trunk fat mass, as measured by dual-energy X-ray absorptiometry, in children aged 3-19 y. Am J Clin Nutr. 2000;72(2):490 495.

20. Jochem C, Leitzmann M. Obesity and Colorectal Cancer. Recent Results Cancer Res. 2016;208:17-41. 
21. Pischon T, Nimptsch K. Obesity and Risk of Cancer: An Introductory Overview. Recent Results Cancer Res. 2016;208:1-15.

22. Nimptsch K, Pischon T. Obesity Biomarkers, Metabolism and Risk of Cancer: An Epidemiological Perspective. Recent Results Cancer Res. 2016;208:199-217.

23. Ito S, Hotta K, Imai K, et al. Preliminary Experience Using FullSpectrum Endoscopy for Colorectal Cancer Screening: Matched Case Controlled Study. Gastroenterol Res Pract. 2016:1349436.

24. Hassan C, Senore C, Radaelli F, et al. Full-spectrum (FUSE) versus standard forward-viewing colonoscopy in an organised colorectal cancer screening programme. Gut. 2016:311906.

25. Imran M, Sayedalamin Z, Alsulami SS, et al. Knowledge and Awareness of Colorectal Cancer among Undergraduate Students at King Abdulaziz University, Jeddah, Saudi Arabia: a Survey-Based Study. Asian Pac J Cancer Prev. 2016;17(5):2479-2483.

26. Zubaidi AM, AlSubaie NM, AlHumaid AA, et al. Public Awareness of Colorectal Cancer in Saudi Arabia: A Survey of 1070 Participants in Riyadh. Saudi J Gastroenterol. 2015;21(2):78-83.

27. Zubaidi AM, AlSubaie NM, AlHumaid AA, et al. Public Awareness of Colorectal Cancer in Saudi Arabia: A Survey of 1070 Participants in Riyadh. Saudi Journal of Gastroenterology: Official Journal of the Saudi Gastroenterology Association. 2015;21(2):78-83.

28. Bukhari H, Mirza M, Almatrafi FG, et al. Public awareness of risk factors, screening and prevention of colorectal cancer. International Journal of Academic Scientific Research. 2016;4(1):135-140. 